\title{
Correlación macroscópica-microscópica en muertes por electrocución: Morgue Central de Lima periodo 2006-2011
}

Macroscopic-microscopic correlation in deaths by electrocution: Central Morgue of Lima 2006-2011

\author{
Denisse Astuhuamán-Canchaya ${ }^{1,2, a, b}$, Kelly Milagritos Casana-Jara ${ }^{1,2, a, d}$, \\ Rosa Elizabeth Carrera-Palao ${ }^{1,2, a, b, c}$, Shermany Francisco Aronés-Guevara ${ }^{1,2, a, b}$, \\ César Andrés Tejada-Valdivia ${ }^{1,2, a, b}$ \\ Instituto de Medicina Legal, Ministerio Público, Perú. \\ ${ }^{2}$ Instituto de Patología, Universidad Nacional Mayor de San Marcos, Lima, Perú \\ ${ }^{a}$ Médico; ${ }^{b}$ Especialidad en Medicina Legal; ${ }^{c}$ Especialidad en Anatomia Patológica; ${ }^{d}$ Residente de Medicina Legal.
}

\begin{abstract}
Resumen
Introducción: La electrocución es una causa de muerte de indole traumática y de alta mortalidad, que produce lesiones y signos externos que deben ser corroborados por hallazgos microscópicos. Objetivo: Correlacionar hallazgos macroscópicos con resultados histopatológicos en muertes por electrocución. Diseño: Descriptivo, transversal, correlacional, retrospectivo. Institución: Instituto Medicina Legal-MP, Instituto Patología, Universidad Nacional Mayor de San Marcos, Lima, Perú. Material: Informes de Necropsia Médicolegal. Intervenciones: En la Morgue Central de Lima, en el periodo 2006-2011, se hizo búsqueda y análisis de información de electrocución. Principales medidas de resultados: Medida de tendencia central, frecuencias y porcentaje. Análisis de correlación. Resultados: De 116 casos, 108 varones y 8 mujeres, la media de edad fue 31,3 años, 23\% fueron electricistas, 18\% trabajaban en construcción. El hecho ocurrió en el centro de trabajo 51\%, con etiología suicida en dos casos. Los hallazgos macroscópicos más frecuentes fueron signos generales de asfixia, lesiones por entrada de corriente eléctrica, hemorragia miocárdica y petequias subpleurales, en $73,3 \%$. Las alteraciones microscópicas fueron: necrosis coagulativa en piel 87 casos, edema cerebral 101 casos, hemorragia pulmonar 83 casos, infarto agudo de miocardio 87 casos, necrosis tubular aguda 72 casos. Al correlacionar los hallazgos macroscópicos y microscópicos más frecuentes, encontramos una asociación significativa (valor $V$ de Cramer de 0,592, p<0,0001). Conclusiones: Los hallazgos macroscópicos más frecuentes (lesión de entrada, hemorragia miocárdica y petequias subpleurales) y los hallazgos microscópicos más frecuentes (necrosis coagulativa en piel, infarto agudo de miocardio y necrosis tubular aguda) relacionados a electrocución parecen estar significativamente asociados.
\end{abstract}

Palabras clave: Electrocución, hallazgos macroscópicos, histopatología.

\section{Abstract}

Introduction: Electrocution is a frequent cause of traumatic death. It produces lesions and external signs that should be corroborated by microscopic findings. Objective: To correlate macroscopic findings with histopathologic results in electrocution deaths. Design: Descriptive, cross-sectional, correlational, retrospective study. Setting: Legal Medicine Institute-MP, Pathology Institute, Universidad Nacional Mayor de San Marcos, Lima, Peru. Material: Medicolegal necropsy reports. Results: From 116 cases, 8 were women and 108 males, mean age was 31.3 years. Twenty three per cent were electricians and $18 \%$ worked in construction. The incident occurred at labor center in $51 \%$. Suicide accounted for two cases. Frequent macroscopic findings were general signs of choking, power input injuries, myocardial hemorrhage and subpleural petechiae in $73.3 \%$. Microscopic alterations were skin coagulative necrosis in 87 cases, cerebral edema in 101, pulmonary hemorrhage in 83, acute myocardial infarction in 87, and acute tubular necrosis in 72 cases. A significant association between macroscopic and microscopic findings ( $0.592 \mathrm{~V}$ Cramer value; $p<0.0001)$ was found. Conclusions: Most common macroscopic findings (lesion in put, myocardial hemorrhage and subpleural petechiae) and most frequent microscopic findings (skin coagulative necrosis, acute myocardial infarction and acute tubular necrosis) related to electrocution seemed to be significantly associated.

Keywords: Electrocution, macroscopic findings, histopathology.

An Fac med. 2014;75(1):31-5 


\section{INTRODUCCIÓN}

La electrocución es la muerte que se presenta por el paso de corriente eléctrica a través del cuerpo humano. El daño que se produce en la persona se debe a que al pasar la corriente eléctrica a través del cuerpo se provoca un calentamiento y una destrucción de los tejidos ${ }^{(1)}$. Es una causa de muerte de índole traumática y de alta mortalidad. Su etiología médico legal en la mayoría de casos es accidental; predomina en varones y en trabajadores de construcción ${ }^{(2)}$. Esta entidad produce en los cadáveres lesiones y signos externos, como son las quemaduras por paso de corriente eléctrica de 'entrada y salida', hallazgos como petequias conjuntivales, cianosis de cara, cuello y lechos ungueales y el chamuscamiento de vellos y cabellos. Dentro de los hallazgos de necropsia más frecuentes se encuentran petequias y/o equimosis subepicárdicas y subpleurales, así como el signo de Piacentino en el piso del IV ventrículo y congestión del resto de los órganos ${ }^{(3)}$, los cuales deben ser corroborados por hallazgos histopatológicos por microscopia, para apoyar de manera fidedigna la causa de muerte por electrocución. Por ello, ambos estudios deben ser complementarios y sus resultados correlacionarse de manera positiva para apoyar el diagnóstico de causa de muerte, sobre todo en casos en los que las lesiones macroscópicas no sean visibles, certeras o acarreen dudas, permitiendo al médico legista poder conocer la frecuencia, características macroscópicas y microscópicas asociadas a las muertes por electrocución y de esta manera tener un panorama más amplio para orientar de manera integral la realización de necropsias en casos donde se sospeche como causa de muerte la electrocución; de esta manera se podrá obtener mayores elementos de prueba para contribuir a una correcta administración de justicia.

Asimismo, no existen investigaciones en nuestro medio que den a conocer las características macroscópicas y microscópicas y la correlación entre estos hallazgos asociados a la muerte por electrocución. Su conocimiento nos permitirá establecer características propias para contribuir a una correcta determinación de la causa de muerte.

El objetivo del presente trabajo fue resaltar la importancia que tienen los estudios histopatológicos para esta entidad y su correlación con los hallazgos macroscópicos. Asimismo, es importante recordar que la necropsia de un cadáver debe de ser completa, metódica, descriptiva y principalmente científica. Será sustentada con todos los medios disponibles para llegar a ello y así contribuir como auxiliares para una buena impartición de justicia.

Es de sumo interés y de gran magnitud social que se estudie y difunda a la electrocución como un fenómeno que se puede prevenir, ya que en la mayoría de casos es de etiología accidental en cualquier grupo de edad, predominando en el adulto joven; en ocasiones llega a ser incapacitante y en el peor de los casos es mortal.

\section{MÉTODOS}

Se realizó un estudio descriptivo, retrospectivo, correlacional de corte transversal, que consistió en la revisión y análisis de los informes de protocolo de necropsia en los que se determinó como causa de muerte electrocución, realizados durante el periodo 20062011 en la División de Tanatología Forense (Morgue Central de Lima) del Instituto de Medicina Legal del Ministerio Público.

El presente trabajo incluye los casos en los cuales durante la necropsia el médico legista solicitó exámenes anatomopatológicos y aquellos casos donde se determinó una data de muerte menor a 24 horas.

Para el cálculo del tamaño muestral se tomó en cuenta un muestreo no probabilístico de conveniencia, tomando la totalidad de casos reportados en el periodo 2006-2011, haciendo un total de 116 informes de protocolo de necropsia estudiados.
La recolección de la información se realizó mediante la aplicación de una ficha de investigación, la cual fue validada por opinión de expertos con un promedio de valoración de $90,5 \%$, en la que se recogió las variables en estudio, se extrajo datos relacionados a filiación, lugar de ocurrencia, etiología médico legal, hallazgos macroscópicos durante la necropsia médico legal y los resultados histopatológicos y quimicotoxicológicos.

Se elaboró una base de datos y se realizó un análisis estadístico descriptivo mediante el cálculo de frecuencias simples, medidas de tendencia central y de dispersión, para cada una de las variables de interés. Se usó el software estadístico SPSS v19.0. Asimismo, se realizó un estudio correlacional con la prueba chi-cuadrado y se calculó un valor de V de Cramer para determinar significancia de correlación.

\section{RESULTADOS}

Se analizó 116 informes de necropsia médico legal realizadas en la División de Tanatología Forense del Instituto de Medicina Legal, durante el periodo enero 2006 a diciembre 2011.

La muestra estuvo constituida por 108 varones y 8 mujeres. La media de edad fue 31,3 años (rango de 01 a 70 años; DE: 15,921 años); 27 (23\%) fueron electricistas y 21 (18\%) trabajaban en construcción civil. El año en el que se presentó mayor cantidad de muertes por electrocución fue el 2009 (25\%). El distrito de procedencia más frecuente fue San Juan de Lurigancho (33,6\%) seguido de Ate Vitarte y Villa el Salvador (tabla 1).

La escena del hecho correspondió al centro de trabajo en 59 casos $(51 \%)$ y al domicilio en 52 casos (45\%). La gran mayoría tuvo etiología médico legal accidental, solo dos casos suicidas.

En la necropsia médico legal se encontró lesiones por entrada de corriente eléctrica en todos los casos, estando ubicadas con mayor frecuencia 
en miembros superiores $(94,8 \%)$; de estas 102 se ubicaron en la mano y 06 en antebrazo. En los dos casos suicidas, las lesiones por entrada se ubicaron en ambas manos.

De las lesiones por entrada de corriente eléctrica en miembros inferiores, solo se presentaron tres casos, en muslo, pierna y pie, respectivamente. Otros segmentos corporales fueron tórax anterior (01), abdomen (01) y pelvis $(01)$.

Lesiones por salida de la corriente eléctrica se encontró en 64 casos $(55,2 \%)$ y fueron más frecuentes en miembro inferior.

De los hallazgos macroscópicos de necropsia, se encontró signos generales de asfixia (cianosis, fluidez sanguínea, hemorragias petequiales en serosas y congestión multivisceral) en todos los casos.

Los hallazgos más frecuentes durante el examen macroscópico de la necropsia médico legal, exceptuando los signos generales de asfixia, fueron: lesiones por entrada de corriente eléctrica, hemorragia miocárdica y petequias subpleurales (tabla 2), los cuales se encontraron simultáneamente en 85 casos $(73,3 \%)$.

Las alteraciones microscópicas encontradas se ubicaron con mayor frecuencia en pulmón, seguidas de cerebro, piel, corazón y riñón, respectivamente. En la descripción microscópica de piel se encontró necrosis coagulativa en 87 casos. En cerebro, 101 casos presentaron edema cerebral y 29 hemorragia subaracnoidea. En pulmón, hemorragia pulmonar y edema pulmonar en 83 y 73 casos, respectivamente.

En 87 casos se halló infarto agudo de miocardio o hemorragia subendocárdica y en 53 casos $(45,7 \%)$ trombosis vascular. En parénquima renal, 72 casos presentaron necrosis tubular aguda.

Se encontró simultáneamente alteraciones histopatológicas en piel, infarto agudo de miocardio y necrosis tubular aguda en 64 casos (55,2\%).
Respecto a la alcoholemia en casos de muertes por electrocución, solo se encontró cinco casos $(4,3 \%$ del total), con cifras comprendidas entre 0,6 g\% y 3,0 g\%. Respecto a la determinación de sustancias tóxicas, todos los resultados fueron negativos.

De los resultados presentados, se aprecia que en todos los casos se halló lesiones por entrada de corriente eléctrica; asimismo todos presentaron signos generales de asfixia. También se encontró alteraciones histopatológicas en el pulmón en todos los casos.

Al analizar la correlación de variables mediante chi cuadrado, hubo asociación entre el hallazgo de hemorragia miocárdica e infarto agudo de miocardio o hemorragia subendocárdi- ca, Xc2 $=98,655(\mathrm{p}<0,0001)$. No hallamos asociación entre el hallazgo de petequias en IV ventrículo y alteraciones histopatológicas en tejido cerebral $X_{c} 2=2,068(p=0,15)$.

$\mathrm{Al}$ correlacionar conjuntamente los hallazgos macroscópicos más frecuentes (lesión de entrada, hemorragia miocárdica y petequias subpleurales) con los hallazgos microscópicos más frecuentes (necrosis coagulativa en piel, infarto agudo de miocardio y necrosis tubular aguda), hubo asociación entre estos, en los casos de muerte por electrocución, $X_{c} 2=40,603(p<0,0001)$. Además, se determinó un valor de $\mathrm{V}$ de Cramer de 0,592 ( $p<0,0001$ ), lo que indica un valor significativo de correlación.

Tabla 1. Variables generales relacionadas a las muertes por electrocución.

\begin{tabular}{|c|c|c|}
\hline \multicolumn{2}{|l|}{ Variables } & Medidas de resumen \\
\hline \multicolumn{3}{|l|}{ Datos generales } \\
\hline \multirow[t]{2}{*}{ Sexo } & Femenino & $8(75,4 \%)^{*}$ \\
\hline & Masculino & $108(24,6 \%)^{*}$ \\
\hline \multicolumn{2}{|l|}{ Edad } & $31,3(\mathrm{DE}:+/-15,9)^{* *}$ \\
\hline \multirow[t]{7}{*}{ Grupos de edad (años) } & 0 a 10 & $11(9,5 \%)^{*}$ \\
\hline & 11 a 20 & $17(14,7 \%)^{*}$ \\
\hline & 21 a 30 & $32(27,6 \%)^{*}$ \\
\hline & 31 a 40 & $26(22,4 \%)^{*}$ \\
\hline & 41 a 50 & $17(14,7 \%)^{*}$ \\
\hline & 51 a 60 & $7(6 \%)^{*}$ \\
\hline & Mayor de 60 años & $6(5,2 \%)^{*}$ \\
\hline \multirow[t]{4}{*}{ Ocupación } & Electricista & $27(27 \%)^{*}$ \\
\hline & Construcción & $21(21 \%)^{*}$ \\
\hline & Cocinero & $11(11 \%)^{*}$ \\
\hline & Otros & $48(48 \%)^{*}$ \\
\hline \multirow[t]{4}{*}{ Distrito de procedencia } & San Juan de Lurigancho & $39(33,6 \%)^{*}$ \\
\hline & Ate Vitarte & $29(25 \%)^{*}$ \\
\hline & Villa El Salvador & $26(22,4 \%)^{*}$ \\
\hline & Otros & $22(19 \%)^{*}$ \\
\hline \multirow[t]{3}{*}{ Lugar de ocurrencia } & Trabajo & $59(51 \%)^{*}$ \\
\hline & Domicilio & $52(45 \%)^{*}$ \\
\hline & Vía pública & $5(4 \%)^{*}$ \\
\hline
\end{tabular}


Tabla 2. Variables relacionadas a hallazgos macroscópicos y microscópicos.

\begin{tabular}{lc} 
Variables & Frecuencia (porcentaje) \\
Hallazgos macroscópicos & \\
\hline Signos generales de asfixia & $116(100 \%)$ \\
Petequias subpleurales & $112(96,5 \%)$ \\
Hemorragia miocárdica & $85(73,3 \%)$ \\
Petequias en IV ventrículo & $60(51,7 \%)$ \\
Lesión por entrada de corriente eléctrica & $100(100 \%)$ \\
Hallazgos microscópicos & \\
Necrosis coagulativa en piel & $87(75 \%)$ \\
Edema cerebral & $77(66,4 \%)$ \\
Edema + Hemorragia pulmonar & $50(43,1 \%)$ \\
Infarto agudo / Hemorragia subendocárdica & $87(75 \%)$ \\
Necrosis tubular aguda & $72(62 \%)$ \\
\hline
\end{tabular}

\section{DISCUSIÓN}

La muerte por electrocución pertenece al grupo de muertes violentas. En nuestro medio no se presenta con mucha frecuencia, ya que representa solo el $2 \%$ del total de las muertes violentas. Sin embargo, es una entidad que en muchas ocasiones pasa desapercibida durante las necropsias médico legales por la escasa información que se obtiene de las actas de levantamiento de cadáver. Por ello es necesario conocer sus principales hallazgos macroscópicos y microscópicos; ambos estudios deben ser complementarios y sus resultados correlacionarse de manera positiva para apoyar el diagnóstico de causa de muerte, sobre todo en casos en los que las lesiones macroscópicas no sean visibles, certeras o acarreen dudas.

Se ha descrito mucho acerca de esta entidad y sus aspectos histopatológicos, tanto en sus lesiones macroscópicas ${ }^{(1,3,4)}$ como microscópicas ${ }^{(3,5,6)}$. En nuestro medio no se cuenta con estudios de esta entidad.

La electrocución es la muerte que se presenta por el paso de corriente eléctrica a través del cuerpo humano; es una de las causas de muerte traumática ${ }^{(7,8)}$.
En 2009, Lederer y col determinaron que la muerte por electrocución es más frecuente en varones jóvenes, entre 20 y 34 años ${ }^{(4,9)}$, profesionales de la electricidad, por contacto directo con la fuente eléctrica y por corrientes de baja tensión, datos semejantes a los obtenidos en el presente estudio.

En 2003, Driscoll y col demostraron que la etiología médico legal de las muertes por electricidad pueden ser accidental, suicida, homicida, homicida y por suplicio ${ }^{(10,11)}$; asimismo, dentro de las accidentales las más frecuentes son las laborales y en el hogar, ocupando el $9^{\circ}$ lugar de las muertes ocurridas por accidentes domésticos, datos idénticos a los obtenidos en nuestro trabajo.

En nuestro estudio, los distritos en los que se presentaron con mayor frecuencia muertes por electrocución fueron San Juan de Lurigancho, Villa el Salvador y Ate Vitarte, que correspondieron a $81 \%$ de casos. Esto podría relacionarse a que en dichos distritos se encuentran la mayor cantidad de grandes empresas industriales, teniendo en cuenta que este tipo de muerte se presenta con mayor frecuencia en electricistas. Estos datos también se asocian a que la mayoría de estas muertes ocurrió en el lugar de trabajo.
En 2005, Knight y col concluyeron que la forma de muerte en la mayor parte de electrocutados se debía a fibrilación ventricular debido al efecto de la corriente en el miocardio, presentando el cadáver palidez facial. También puede producirse la muerte por parálisis respiratoria, tetanización del diafragma y de los músculos intercostales mostrando el cadáver facies cianótica ${ }^{(12-15)}$, lo que se relaciona con los hallazgos obtenidos en nuestro estudio, pues fue hallazgo histopatológico el infarto agudo en $75 \%$ de los casos.

El trayecto de la corriente eléctrica a través del cuerpo determina la gravedad de las lesiones; el paso por el tórax se asocia con una mortalidad del $60 \%$. Sellier ${ }^{(16)}$ señala como trayecto más peligroso el que discurre desde el brazo izquierdo hasta los pies, mientras Alexander apunta que es más letal el que recorre las dos extremidades superiores que el que viaja de una extremidad superior a una inferior. Para Di Maio y Dana ${ }^{(17)}$, el riesgo de muerte es máximo cuando interesa el corazón o el cerebro. En nuestro estudio se encontró lesiones por entrada de corriente eléctrica en todos los casos, estando ubicadas con mayor frecuencia en miembros superiores (94,8\%). Asimismo, en los dos casos suicidas las lesiones por entrada se ubicaron en ambas manos, datos que se relacionan con lo indicado por Alexander en cuanto a la mayor letalidad de la electrocución con respecto al trayecto de la corriente eléctrica a través de los miembros superiores ${ }^{(10)}$.

Lindström y col, en un estudio realizado en muertes por electrocución, determinaron resultados analíticos que revelaron, en $20 \%$ de los casos, la presencia de alcohol en sangre ${ }^{(1)}$, datos que difieren a los encontrados en nuestro estudio, en el que solo hubo cinco casos $(4,3 \%$ del total), con valores entre $0,6 \mathrm{~g} \%$ y $3,0 \mathrm{~g} \%$ de alcoholemia. Respecto a la determinación de sustancias tóxicas, todos los resultados fueron negativos.

Knight en 1996, Mason en 2000 y Di Maio en 2001, anglo americanos, 
no consideraron importantes las hemorragias del romboencéfalo ${ }^{(15,18)}$. En nuestro estudio encontramos macroscópicamente petequias a nivel del IV ventrículo -signo de Piacentino- en $51,7 \%$, dato que difiere con los estudios mencionados. Sin embargo, al analizar la correlación de variables mediante chi cuadrado no encontramos asociación entre el hallazgo de petequias en IV ventrículo y alteraciones histopatológicas en tejido cerebral, ya que el hallazgo histopatológico más frecuente en cerebro fue el edema.

Al correlacionar los hallazgos macroscópicos más frecuentes (lesión de entrada, hemorragia miocárdica y petequias subpleurales) con los hallazgos microscópicos más frecuentes (necrosis coagulativa en piel, infarto agudo de miocardio y necrosis tubular aguda), hallamos asociación en los casos de muerte por electrocución. Además, se determinó un valor de $\mathrm{V}$ de Cramer de $0,592$ ( $p<0,0001)$, lo que indica un valor significativo de correlación.

Por lo tanto, podemos concluir que para poder determinar electrocución como causa de muerte, son determinantes los hallazgos macroscópicos. Sin embargo, estos tienen que ser corroborados por los estudios histopatológicos a la microscopia, sobre todo en aquellos casos donde las lesiones o signos externos no sean concluyentes o no estén lo suficientemente claros.
Este trabajo es un antecedente para la realización de posteriores investigaciones de muertes por electrocución, ya que se cuenta con la infraestructura y los recursos humanos para este fin. La literatura en la que se basa el trabajo es de origen extranjero, ya que en nuestro medio no existe publicación sobre esta entidad. Por ello se propone realizar una profunda investigación de los aspectos histopatológicos de la electrocución por parte del personal médico del Instituto de Medicina Legal; pues es una entidad no muy frecuente, pero de gran dificultad en cuanto al diagnóstico, si no se tiene la información adecuada.

\section{REFERENCIAS BIBLIOGRÁFICAS}

1. Patitó JA. Tratado de Medicina Legal y elementos de patologia forense. Argentina: Editorial Quórum. 2003;621 pp.

2. Mileaf H. Electricidad. 1 edición. México: Editorial Limusa. 2000:113 pp.

3. Calabuig G. Medicina Legal y Toxicología. $6^{\text {a }}$ Ed. Barcelona, España: Masson. 2005: 419 pp.

4. Maldonado SM; Martínez Garcia P. Muerte po electrocución. Cuadernos de Medicina Forense. NO 32-Abril. 2003. España 2003:71-4.

5. Robbins. Patologia Estructural y Funcional. $6^{\text {a }}$ Ed Mc-Graw-Hill- Interamericana. 2000:460 pp.

6. Wang Y, Liu M, Cheng W-B, et al. Endothelial cell membrane perforation of aorta and pulmonary artery in the electrocution victims. Forensic Sci Intern. 2008:178(2-3):204-6.

7. Bailey B, Forget S, Gaudreault P. Prevalence of potential risk factors in victims of electrocution. Forensic Sci Int. 2001;123(1):58-62.

8. Vargas A. Medicina Legal. $3^{\text {a }}$ Ed. Costa Rica: Lehmann editores. 1983:197-9.
9. Ashesh GW, Dinesh RS. Electrocution by metal kite line. Forensic Sci Intern. 2006;163 (6):141-3.

10. Chan P, Duflou J. Suicidal electrocution in Sydney: a 10-year case review. J Forensic Science. 2008;53(2):455- 9 .

11. Lindström R, Bylund PO, Eriksson A. Accidental deaths caused by electricity in Sweden, 19752000. J Forensic Sci. 2006; 51:1383-8.

12. James TN. Cardiac abnormalities demonstrated postmortem in four cases of accidental electrocution and their potential significance relative to nonfatal electrical injures of de heart. Am Heart J. 1990;120(1):143-55.

13. Spies C, Trohman RG. Narrative review: electrocution and life-threatening electrical Injures". Ann Internal Med. 2006;145(7):531.

14. Karch FV, D'errico SV. Cardiac pathology in death from electrocution. Int J Leg Med. 2006;120:7982.

15. Knigth B. Lesiones por electricidad. En: Knigth B Medicina Forense de Simpson. $2^{\circ}$ edición. México: Manual Moderno. 1999:180-2.

16. Sellier JE. Schäden und Tod durch Elecktrizität. En: Mueller B, editor. Gerichtliche medizin, Teil 1,2. Berlin/Heidelberg/New York: Springer; 1975:538-60.

17. Di Maio VJM, Dana SE. Electrocución. En: Manual de patologia forense. Madrid: Diaz de Santos S.A.; 2003;207-16.

18. Di Maio V. Forensic Pathology. $2^{\text {a }}$ ed. CRC Press 2001;423-9.

Trabajo presentado a las XII Jornadas Científicas Sanfernandinas 2013, XV Jornadas de Investigación en Salud, XXII Jornadas Sanfernandinas Estudiantiles, Facultad de Medicina, Universidad Nacional Mayor de San Marcos, agosto 2013.

Correspondencia:

Denisse Astuhuamán Canchaya

Correo electrónico: densac20@hotmail.com 